This item was submitted to Loughborough's Research Repository by the author.

Items in Figshare are protected by copyright, with all rights reserved, unless otherwise indicated.

\title{
Estimation of traffic densities for multilane roadways using a Markov model approach
}

PLEASE CITE THE PUBLISHED VERSION

http://dx.doi.org/10.1109/TIE.2011.2180271

PUBLISHER

(C) IEEE

VERSION

AM (Accepted Manuscript)

LICENCE

CC BY-NC-ND 4.0

REPOSITORY RECORD

Singh, Karandeep, and Baibing Li. 2019. "Estimation of Traffic Densities for Multilane Roadways Using a Markov Model Approach”. figshare. https://hdl.handle.net/2134/12189. 
This item was submitted to Loughborough's Institutional Repository (https://dspace.lboro.ac.uk/) by the author and is made available under the following Creative Commons Licence conditions.

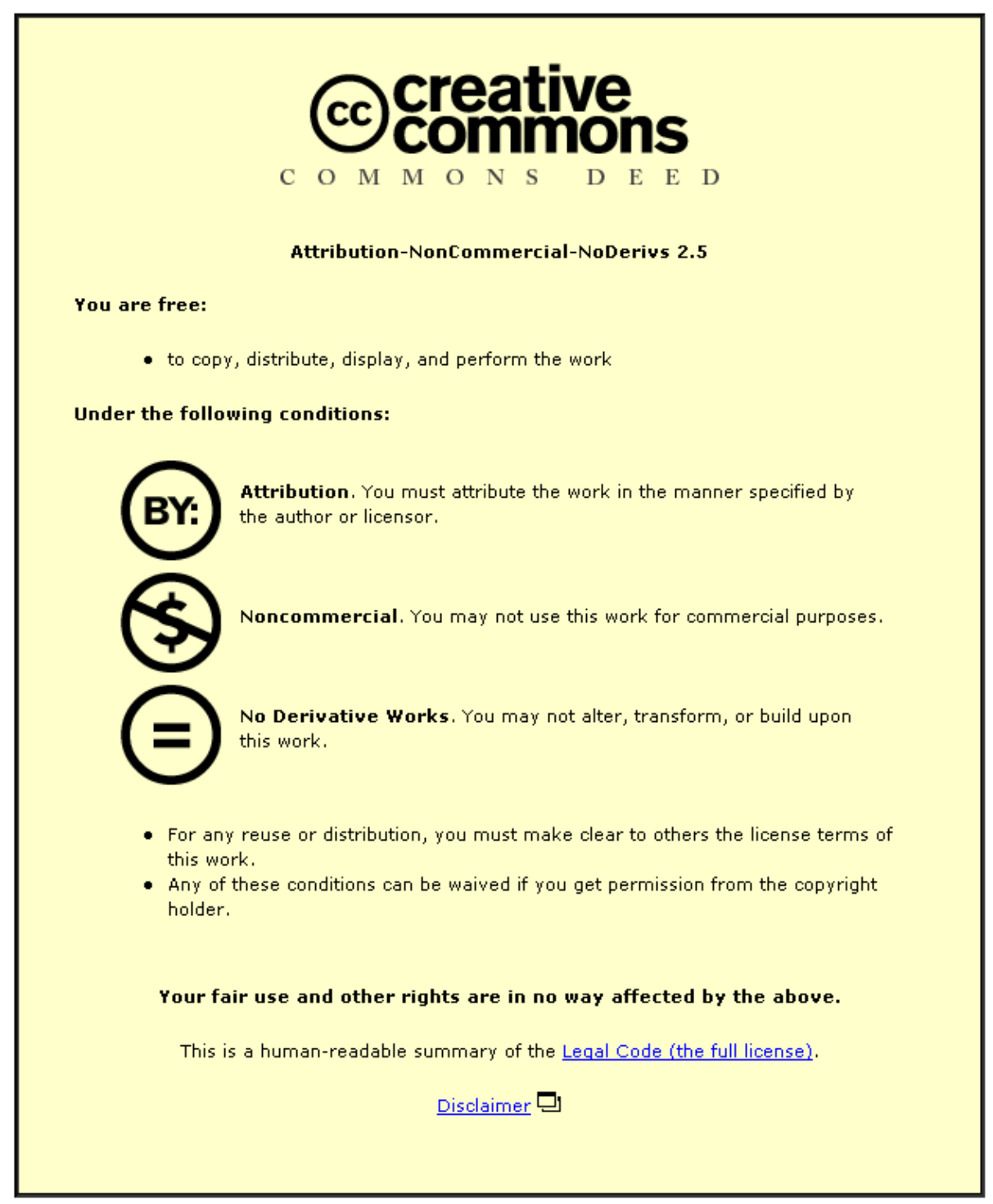

For the full text of this licence, please go to: http://creativecommons.org/licenses/by-nc-nd/2.5/ 


\title{
Estimation of Traffic Densities for Multi-Lane Roadways Using a Markov Model Approach
}

\author{
Karandeep Singh and Baibing Li
}

\begin{abstract}
Inductive loop detectors are widely deployed in strategic roadway networks. This paper investigates recursive estimation of traffic densities using the information provided by loop detectors. The existing studies for multi-lane roadways mainly focus on the scenario where vehicles' lane change movements are not common and can be ignored. This research, however, takes into consideration of lane change effect in traffic modeling and incorporates a Markov chain into the state space model to describe the lane-change behavior. We update the traffic density estimate using the Kalman filter. To avoid the approximation due to the linearization of the nonlinear observation equation in the extended Kalman filter, we have considered a suitable transformation. Numerical studies were carried out to investigate the performance of the developed approach. It is shown that it outperforms the existing methods.
\end{abstract}

Index Terms - Density estimation, Kalman filter, lane change, Markov chain process.

\section{INTRODUCTION}

$\mathrm{I}^{\mathrm{N}}$ $\mathrm{N}$ both developed and emerging-economy countries, major cities continue to experience increasing traffic congestion. In recent years, intelligent transportation systems are shown to be a promising approach to help manage congested traffic. These systems fuse traffic-surveillance-related information from a variety of sensors deployed across traffic networks.

An intelligent transportation system requires real-time information to make effective control decisions and to deliver trustworthy information to users, such as travel time, congestion level, etc. There are three fundamental inputs required by intelligent transportation systems, i.e. traffic volume, vehicular speed, and traffic density.

In practice, point sensors such as inductive loop detectors are widely used for traffic detection. These point sensors can continuously count vehicle numbers in traffic streams passing at the sensor locations (see e.g. [1], [2]) so the information on traffic volume is directly available from a point sensor. In addition, vehicular speed can be calculated using the information collected from either a single loop detector [2] or

Copyright (c) 2011 IEEE. Personal use of this material is permitted. However, permission to use this material for any other purposes must be obtained from the IEEE by sending a request to pubs-permissions@ieee.org.

Karandeep Singh is with the School of Business \& Economics, Loughborough University, Loughborough, LE11 3TU UK (e-mail: k.singh@lboro.ac.uk).

Baibing $\mathrm{Li}$ is with the School of Business \& Economics, Loughborough University, Loughborough, LE11 3TU UK (corresponding author. Tel. +441509-228841. e-mail: b.li2@lboro.ac.uk). a speed trap [3]. Hence, speed information can also be derived from a point sensor.

Traffic density defined as the number of vehicles in a lane within a unit length of roadway segment, however, cannot be measured directly using point sensors. As an instantaneous and range concept, traffic density can be worked out using a snapshot photo of traffic by an aerial camera along a stretch of roadway (see e.g. [4], [5]). Practical traffic network systems, particularly freeway networks, however, do not have aerial cameras to continuously monitor the traffic in real time. Although dense point sensor systems (e.g. inductive loop detectors) could approximate continuous measurements in space, the cost would be prohibitive in general. Consequently the problem of estimating the number of vehicles traversing within a roadway segment presents an important issue and has attracted a great deal of research in the recent decades.

The Kalman filter is an efficient recursive technique to update the estimated state vector of a dynamic system from a series of noisy measurements. It is widely used in the studies on intelligent transportation systems. The applications include the navigation filter in the GPS receiver for car navigation systems [6], image processing and behavior planning for intelligent vehicles [7], advanced parking assistance systems [8], the estimation of lane width in a vehicle lane tracking system [9], and the modeling of vehicular energy storage [10]. Recently it has also been applied to the area other than state estimation such as fault detection [20] and optimization [21].

A pioneering study on traffic density estimation using Kalman filter can be traced back to the early work in [11] in 1970s. Since then the Kalman filter has been employed quite frequently in the literature to estimate roadway traffic densities, including linear Kalman filter [15], extended Kalman filter [12][13], mixture Kalman filter [14], etc.

This paper is mainly based on the recent research framework developed in [12]. It investigates traffic flow modeling via the approach of state space model, and the application of Kalman filtering to roadway traffic density estimation. Unlike [12] where lane change behavior is ignored, however, we will take into account of lane change effect in the analysis and will model lane change behavior directly in the state equation of the state space model. Lane change behavior can have great impact on traffic flow for some roadway sections and it has attracted considerable attention in the traffic literature. However, although lane change behavior is an important aspect of transport research, it has not been studied much in relation with density estimation 
[16]. In addition, some of the lane change models assume accurate measurements of traffic counts (e.g. [17]) but this is not true in practice because the operation of conventional inductive loop detectors involves substantial measurement errors (see e.g. [12]). In this paper we will use a Markov chain process to describe lane change behavior.

Now we turn to the issue of the estimation of traffic densities. In most existing studies that use state space models to estimate traffic densities, the speed-density relationship serves as the observation equation. As the speed-density relationship in the Drake's model [18] is nonlinear, the existing methods utilize the extended Kalman filter to update the estimated traffic state vector for online traffic surveillance. We note, however, the extended Kalman filter involves an approximation where the nonlinear system is linearized. This can sometimes lead to substantial estimation errors (see e.g. [2]). To avoid the linearization in the extended Kalman filter used in the existing methods, we have considered a transformation in this paper. As demonstrated later, the accuracy of traffic density estimation can be substantially improved using this transformation.

This paper is organized as follows. In the next section we will develop a model for traffic flow where lane change effect is taken into account. Then we consider a transformation approach to transform the observation equation from a nonlinear equation to a linear form. The Kalman filter technique is then applied to update the estimated traffic densities. Sections III and IV are devoted to two simulation studies where the performance of the developed state space model is compared with the existing method in [12]. Then the developed approach is illustrated using real traffic data. Finally discussion and conclusions are offered in Section VI.

\section{METHODOLOGY}

Effective real time surveillance and control of roadway traffic rely on the information regarding the numbers of vehicles in different segments of multi-lane roadways. We consider a roadway section with $N$ different segments and $M$ different lanes, where each segment is defined to be a detection zone with one upstream and one downstream embedded inductive loop detector, plus a speed sensor. Following [12] and [13], we assume that the detectors are deployed about 500 meters to 1,500 meters apart. Although longer segments do not cause any technical difficulties for the approach in this paper, traffic condition may change substantially and thus may lead to unreliable results.

We will investigate the estimation of traffic density in each lane of each roadway segment using traffic measurements from the inductive loop detectors.

\section{A. A Brief Summary of the Existing Models}

First, we briefly summarize a typical state space model used in the literature for traffic density estimation.

Let $\mathbf{x}(t)=\left[x_{11}(t), x_{12}(t), \ldots, x_{M N}(t)\right]^{T}$ denote the state vector where $x_{i j}(t)$ is the number of vehicles in lane $j$ of segment $i$ in time interval $t(t=0,1,2, \ldots)$. The state equation in the existing studies is usually formulated on the basis of traffic conservation equation, where the number of vehicles in each lane $j$ of a segment $i$ is equal to the number of vehicles in the previous time interval, plus the net gain of vehicles entering and leaving the upstream and downstream loop detectors respectively (see e.g. [12]):

$$
x_{i j}(t+1)=x_{i j}(t)+u_{i j}(t)-\tilde{u}_{i j}(t)+\varepsilon_{i j}(t)-\tilde{\varepsilon}_{i j}(t),
$$

where $u_{i j}(t)$ and $\tilde{u}_{i j}(t)$ denote the counts of vehicles entering and leaving the upstream and downstream detectors in lane $j$ of segment $i$ in time interval $t . \varepsilon_{i j}(t)$ and $\tilde{\varepsilon}_{i j}(t)$ denote the corresponding counting errors. The above state equation can be rewritten in a matrix form:

$$
\mathbf{x}(t+1)=\mathbf{x}(t)+\mathbf{w}(t)+\xi(t),
$$

where vector $\mathbf{w}(t)$ includes the entries of the net gain $u_{i j}(t)-\tilde{u}_{i j}(t)$, and $\boldsymbol{\xi}(t)$ includes the entries $\varepsilon_{i j}(t)-\tilde{\varepsilon}_{i j}(t)$ for all $i$ and $j$. Let $\mathbf{Q}$ denote the covariance matrix of $\boldsymbol{\xi}(t)$.

Following [12], we assume that the counting errors $\varepsilon_{i j}(t)$ and $\tilde{\varepsilon}_{i j}(t)$ are independent of each other, having an identical distribution with zero mean and variance $\sigma^{2}$. The entries of $\mathbf{Q}$ can be worked out as follows. For any $i$ and $j$, we have $\operatorname{var}\left(x_{i j}(t)\right)=2 \sigma^{2}$. In addition, for any lane $j$, since the number of vehicles leaving a segment $i$ is equal to the number of vehicles entering the neighboring segment $i+1$, we have $\operatorname{cov}\left(x_{i j}(t), x_{(i+1) j}(t)\right)=-\sigma^{2}$. All the other entries of matrix $\mathbf{Q}$ are equal to zero due to the independence assumption.

In traffic engineering, the relationship between traffic speed $v$ and density $K$ is given by (see [18]):

$$
v=v^{*} \exp \left[-\frac{1}{2}\left(\frac{K}{K^{*}}\right)^{2}\right],
$$

where $v^{*}$ is the free flow speed and $K^{*}$ is the density corresponding to the maximum flow in a lane of a roadway segment. This relationship forms the basis of the observation equation in the existing studies such as [12]. Specifically, by taking into account of noise, the speed measurement in lane $j$ of segment $i$ is assumed to satisfy the following equation:

$$
v_{i j}(t)=v_{i j}^{*} \exp \left[-0.5 \theta_{i j}\left(\frac{x_{i j}(t)}{K_{i j}^{*} L_{i}}\right)^{2}\right]+\eta_{i j}(t),
$$

where $L_{i}$ is the length of segment $i . \eta_{i j}(t)$ is the corresponding measurement error. All measurement errors are assumed to be independent of each other with a common variance $\tau^{2}$ (see [12]). The parameters $v_{i j}^{*}$ and $K_{i j}^{*}$ are treated as tuning parameters in [12]. In this paper, we assume that the relationship between traffic speed and density is calibrated using a coefficient $\theta_{i j}$.

Let $\mathbf{h}(\mathbf{x}(t))$ denote the vector of the nonlinear functions given by the first term of the right-hand-side of equation (3). 
The observation equation may be rewritten in a matrix form

$$
\mathbf{v}(t)=\mathbf{h}(\mathbf{x}(\mathbf{t}))+\boldsymbol{\eta}(t),
$$

where vector $\boldsymbol{\eta}(t)$ includes the entries $\eta_{i j}(t)$ for all $i$ and $j$. $\mathbf{R}=\tau^{2} \mathbf{I}$ is the covariance matrix of $\boldsymbol{\eta}(t)$ with $\mathbf{I}$ an identity matrix. Similar state space models were also incorporated in other studies such as [13].

\section{B. A Markov Chain Process for Lane Change Behavior}

In equation (1), lane change behavior is completely ignored. This may not be realistic for some roadway segments. In this subsection, we will extend the above state equation so that lane change behavior is modeled explicitly and the dynamic of traffic flow is better represented by the state equation.

We first focus on the scenario of single roadway segment. In this paper, the maneuver of a vehicle in a roadway segment is modeled as a Markov chain process where each lane is characterized as a state of the process.

Specifically, we assume that each vehicle in the roadway segment has a certain probability to stay in the current state (lane), or to change from one state (lane) to another. We also assume that the traffic flow is stable so that the transition probabilities for the lane change behavior retain approximately constant over time. In practice, the time period of interest (say a day) may be split into several sub-periods so that traffic flow is stable within each sub-period. Now let $p_{j k}$ denote the transition probability that a vehicle moves from state (lane) $j$ to state (lane) $k$.

With the lane change behavior being taken into consideration, the numbers of vehicles entering/leaving a lane from/to the neighboring lane(s) must be included into the state equation. For ease of exposition, we consider a 3-lane problem first. For the middle lane $j$ of segment $i$, the state equation has now become to

$$
\begin{aligned}
x_{i j}(t+1)= & p_{j j} x_{i j}(t)+p_{(j-1) j} x_{i(j-1)}(t)+p_{(j+1) j} x_{i(j+1)}(t) \\
& -p_{j(j-1)} x_{i j}(t)-p_{j(j+1)} x_{i j}(t) \\
& +u_{i j}(t)-\tilde{u}_{i j}(t)+\varepsilon_{i j}(t)-\tilde{\varepsilon}_{i j}(t) .
\end{aligned}
$$

Likewise, for the outermost lane $j=1$, the state equation is

$$
\begin{gathered}
x_{i j}(t+1)=p_{j j} x_{i j}(t)+p_{(j+1) j} x_{i(j+1)}(t)-p_{j(j+1)} x_{i j}(t) \\
+u_{i j}(t)-\tilde{u}_{i j}(t)+\varepsilon_{i j}(t)-\tilde{\varepsilon}_{i j}(t) .
\end{gathered}
$$

For the innermost lane, we have a similar equation.

Now we extend the state equation to an $M$-lane and $N$ segment roadway. First define the system matrix $\mathbf{A}_{n}=\left[a_{j k}^{(n)}\right]$ for the $n$th roadway segment via the transition probabilities:

$$
a_{j k}=\left\{\begin{array}{ll}
p_{k j} & \text { if } j \neq k \\
1-\sum_{l \neq j} p_{j l} & \text { if } j=k
\end{array},\right.
$$

where to simplify notation, we have suppressed the superscript roadway segment $n$ in the above definition.

The state equation can thus be written as follows:

$$
\mathbf{x}(t+1)=\mathbf{A x}(t)+\mathbf{w}(t)+\xi(t)
$$

where the system matrix is $\mathbf{A}=\operatorname{diag}\left\{\mathbf{A}_{1}, \ldots, \mathbf{A}_{N}\right\}$.

\section{Density Estimation}

The estimate of the traffic density vector can be updated on the basis of the nonlinear state space model (4) and (5). The extended Kalman filter allows the system to be nonlinear. During each time interval, the nonlinear system is linearized about the current estimate of the state vector.

Specifically, let $\hat{\mathbf{x}}(t \mid t)$ denote the estimate of the state vector in each time interval $t$. Then for equation (4), the Jacobian evaluated at the current estimate $\widehat{\mathbf{x}}(t \mid t)$ is

$$
\mathbf{H}(t)=\partial \mathbf{h}(\mathbf{x}) /\left.\partial \mathbf{x}\right|_{\mathbf{x}=\hat{\mathbf{x}}(t \mid t)} .
$$

By some algebra it can be shown that matrix $\mathbf{H}(t)$ is diagonal with each diagonal entry equal to $\frac{-\theta_{i j} v_{i j}(t) x_{i j}(t)}{\left(K_{i j}^{*} L_{i}\right)^{2}}$. The observation equation can thus be approximated as

$$
\mathbf{v}(t) \approx \mathbf{h}(\hat{\mathbf{x}}(t \mid t))+\mathbf{H}(t)[\mathbf{x}(t)-\hat{\mathbf{x}}(t \mid t)]+\boldsymbol{\eta}(t) .
$$

Let $\hat{\mathbf{x}}(t+1 \mid t)$ and $\mathbf{P}(t+1 \mid t)$ denote the one-step forecast of the state vector and the corresponding covariance matrix in each time interval $t$. In addition, let $\mathbf{P}(t \mid t)$ denote the covariance matrix of the estimated state vector in each time interval $t$. By applying the extended Kalman filter, the traffic density vector of a multi-lane multi-segment roadway can be estimated using the following steps (see e.g. [12], [19]):

\section{1) Initialization:}

$$
\hat{\mathbf{x}}(0 \mid 0)=\mathbf{x}_{0}, \mathbf{P}(0 \mid 0)=\mathbf{P}_{0} \text {, and } t=0 \text {. }
$$

2) One-step forecast of the state vector:

$$
\begin{gathered}
\hat{\mathbf{x}}(t+1 \mid t)=\mathbf{A} \hat{\mathbf{x}}(t \mid t)+\mathbf{w}(t), \\
\mathbf{P}(t+1 \mid t)=\mathbf{A P}(t+1 \mid t) \mathbf{A}^{T}+\mathbf{Q}
\end{gathered}
$$

3) Compute the Kalman gain matrix:

$$
\mathbf{M}=\mathbf{P}(t+1 \mid t) \mathbf{H}(t)\left[\mathbf{H}(t) \mathbf{P}(t+1 \mid t) \mathbf{H}(t)^{T}+\mathbf{R}\right]^{-1} .
$$

4) Update the estimate and its covariance matrix:

$$
\begin{gathered}
\hat{\mathbf{x}}(t+1 \mid t+1)=\hat{\mathbf{x}}(t+1 \mid t)+\mathbf{M}[\mathbf{v}(t)-\mathbf{h}(\hat{\mathbf{x}}(t+1 \mid t))], \\
\mathbf{P}(t+1 \mid t+1)=\mathbf{P}(t+1 \mid t)-\mathbf{M H}(t) \mathbf{P}(t+1 \mid t) .
\end{gathered}
$$

5) Let $t=t+1$ and return to step 2 .

\section{The Transformed Observation Equation}

As seen in the previous section, observation equation (3) for the traffic density is nonlinear. Consequently, to apply the Kalman filtering technique, it needs to be linearized, as shown in equation (6). Such linearization can sometimes produce un- 
reliable results where the estimates of the state variables were totally off the track (see e.g. [2]). In this subsection, we investigate a transformation approach that transforms the observation equation from a nonlinear form to a linear one so that the approximation is avoided. Specifically, we define a transformed observation as follows:

$$
z_{i j}(t)=\left\{\log \left[v_{i j}^{*} / v_{i j}(t)\right]\right\}^{1 / 2} .
$$

It is the square root of the difference in speed measured on logarithmic scale between the free flow and the current traffic flow. From equation (2), it is straightforward to obtain $z_{i j}(t)=\left[\theta_{i j} /\left(\sqrt{2} K_{i j}^{*} L_{i}\right)\right] x_{i j}(t)$ with a coefficient $\theta_{i j}$ added for the calibration purposes. By taking into account of noise, the transformed observation equation can be written as

$$
z_{i j}(t)=\left[\theta_{i j} /\left(\sqrt{2} K_{i j}^{*} L_{i}\right)\right] x_{i j}(t)+e_{i j}(t),
$$

where the noise $e_{i j}(t)$ is assumed to be independent of each other, having a zero mean and a variance $\tilde{\tau}^{2}$. Now let $\mathbf{G}$ be a diagonal matrix with diagonal entries equal to $\theta_{i j} /\left(\sqrt{2} K_{i j}^{*} L_{i}\right)$. The observation equation can be written in a matrix form:

$$
\mathbf{z}(t)=\mathbf{G}(t) \mathbf{x}(t)+\mathbf{e}(t),
$$

where $\mathbf{z}(t)$ and $\mathbf{e}(t)$ are vectors with entries $z_{i j}(t)$ and $e_{i j}(t)$ respectively. The covariance matrix of $\mathbf{e}(t)$ is $\widetilde{\mathbf{R}}=\tilde{\tau}^{2} \mathbf{I}$.

Now since both state equation (5) and observation equation (8) are linear, the linear Kalman filter can be applied. Specifically, steps 1 to 4 in the above extended Kalman filter algorithm retain the same form but $\mathbf{H}(t)$ is now replaced with $\mathbf{G}(t)$. In addition, the estimated state vector is updated as:

$$
\begin{aligned}
\hat{\mathbf{x}}(t+1 \mid t+1)= & \hat{\mathbf{x}}(t+1 \mid t) \\
& +\mathbf{M}[\mathbf{z}(t)-\mathbf{G}(t) \hat{\mathbf{x}}(t+1 \mid t)] .
\end{aligned}
$$

The advantage of this approach is that the approximation in equation (6) caused by the linearization is avoided.

In practical online applications, the state covariance matrix $\mathbf{P}(t \mid t)$ may become asymmetrical and/or have negative eigenvalues due to rounding error. In addition, the matrix $\mathbf{H}(t) \mathbf{P}(t+1 \mid t) \mathbf{H}(t)^{T}+\mathbf{R}$ may become singular and thus cause problem when calculating matrix $\mathbf{M}$. These issues are normally addressed in the implementation via the Cholesky factorization or singular value decomposition algorithms. Simon [19] summarizes some widely implemented solutions for these stability issues.

\section{SIMULATION FOR LANE CHANGE EFFECT}

In this section, state equation (5) for the estimation of traffic densities will be tested via simulation. One major advantage of simulation study is that the 'true' values of traffic densities are known a priori so that it is straightforward to assess the performance of the developed approach in terms of accuracy.
When comparing the developed state equation (5) with state equation (1) used in [12], we will use the same observation equation (3) so that we can focus on lane change effect.

\section{A. Simulation Description}

We considered a simulation scenario of a 3-lane roadway with a single segment and the time period of 2 hours. The length of the roadway segment $L_{i}(i=1,2,3)$ was set equal to 400 meters long. It was assumed that the upstream and downstream loop detector stations measured traffic every 20s which was taken as the duration of the time intervals in the algorithm. This resulted in a total of 360 time steps. Following [12], the density $K_{i j}^{*}$ corresponding to the maximum flow was set equal to 32, the free speed $v_{i j}^{*}$ was set to be $104.76 \mathrm{~km} / \mathrm{h}$, and the calibration coefficient $\theta_{i j}$ was set equal to 1 . The initial numbers of vehicles in the simulation was set equal to $0.5 L_{i} K_{i j}^{*}$. The system matrix was set to be

$$
\mathbf{A}=\left[\begin{array}{ccc}
0.35 & 0.25 & 0 \\
0.65 & 0.55 & 0.45 \\
0 & 0.20 & 0.55
\end{array}\right],
$$

where it was assumed that no lane change was possible from the innermost lane to the outermost lane or verse visa as the time intervals are short. Clearly the middle lane is the most affected lane due to vehicles' movements between lanes and we will focus on this lane in the subsequent analysis.

The real time traffic density in each lane was simulated using equation (5) where the counts of vehicles entering and leaving the segment, $u_{i j}(t)$ and $\tilde{u}_{i j}(t)$, were simulated as Poisson variates with a mean $\lambda$. The error terms $\varepsilon_{i j}(t)$ and $\tilde{\varepsilon}_{i j}(t)$ were simulated as normal variates with zero mean and variance $\sigma^{2}$. The speed measurements were simulated using equation (3) where $\eta_{i j}(t)$ was simulated as normal variates with zero mean and variance $\tau^{2}$. The settings of the parameters, $\lambda, \sigma$ and $\tau$, varied from experiment to experiment in the simulation below to reflect different scenarios.

Each experiment was repeated 100 times. The evaluation of method was based on the Root Mean Square Error (RMSE) between the 'true' and estimated vehicle counts.

\section{B. Simulation Results}

First, we set $\sigma=1, \tau=2$ and $\lambda=10$ in the simulation. This represents the scenario where on average there were $\lambda=10$ vehicles entering the upstream detector and leaving the downstream detector in each lane of the roadway segment in every 20s time interval. The measurement error of the loop detectors was about $\sigma=1$ vehicle per time interval, and the measurement error of the speed detectors was about $\tau=2 \mathrm{~km} / \mathrm{h}$.

Fig. 1 (upper) displays the 'true' vehicle counts (broken line) and the estimated values (real line) in the middle lane obtained using the developed state equation (5) in one run of the experiment. 

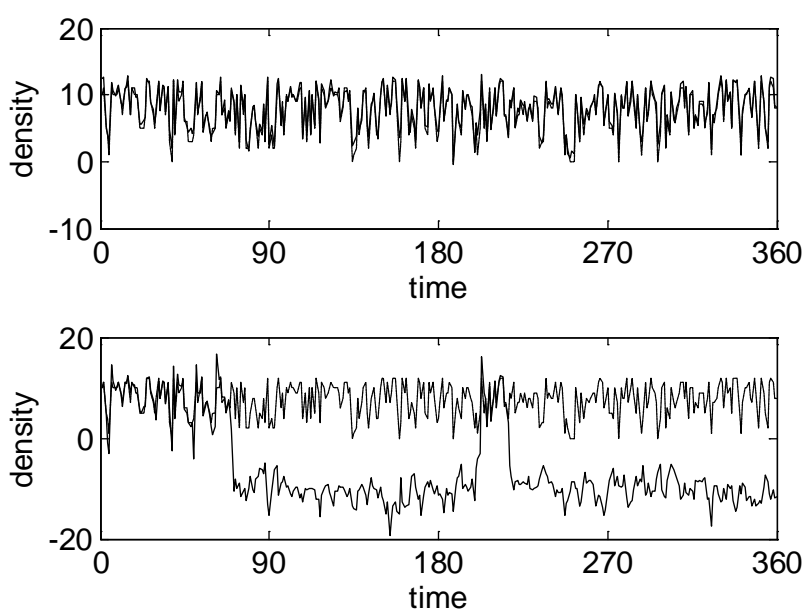

Fig. 1. The 'true' vehicle counts in the middle lane (broken line) and the estimated values (real line) obtained using equation (5) (upper graph) and obtained using the model in [12] (lower graph).

Overall, Fig. 1 (upper) shows that the developed state equation (5) performed well: the estimated vehicle counts were fairly close to the 'true' vehicle counts.

Next, we investigate the performance of the developed state equation in various scenarios where the parameters $\tau$ and $\sigma$ were set at different levels: $\tau=1,3$ and $\sigma=1,2,3$. They represent different magnitudes of the error terms. For each scenario, the experiment was repeated 100 times. The resulting RMSEs averaged over the repeated 100 runs are displayed in Table I.

Table I

Average RMSEs over 100 simulation runs for a three-lane roadway using the developed state equation (5) with $\lambda=10$

\begin{tabular}{ccccccc}
\hline & \multicolumn{3}{c}{$\tau=1$} & & & $\tau=3$ \\
\hline$\sigma$ & Lane 1 & Lane 2 & Lane 3 & Lane 1 & Lane 2 & Lane 3 \\
\hline 1 & 1.01 & 0.91 & 1.00 & 1.06 & 1.05 & 1.06 \\
2 & 1.21 & 1.01 & 1.19 & 0.97 & 0.90 & 0.98 \\
3 & 1.40 & 1.18 & 1.36 & 1.01 & 0.91 & 1.01 \\
\hline
\end{tabular}

Overall it can be seen from Table I that the estimation errors are low: on average it was about one vehicle per time interval.

\section{Comparison with the Existing Method}

Next, we compare the performance of the state equation (5) with that used in [12]. As mentioned earlier, the model considered in [12], i.e. equation (1), ignores lane change effect. Hence it works well only if few vehicles make lane changes. Consequently when there are a substantial number of vehicles that change their lanes, state equation (1) fails to reflect the true dynamic of traffic flow, and thus we expect the model has a poorer performance.

Fig. 1 (lower) displays the 'true' vehicle counts in the middle lane (broken line) and the corresponding estimated values (real line) based on state equation (1) in one run of the experiment. It can be seen that the quality of the estimates was poor: the impact of lane change was substantial and consequently the estimated density greatly departed from the ‘true' values.

Table II

Average RMSEs over 100 simulation runs for a three-lane roadway using the state equation in [12] with $\lambda=10$

\begin{tabular}{ccccccc}
\hline & \multicolumn{3}{c}{$\tau=1$} & & \multicolumn{3}{c}{$\tau=3$} \\
\hline$\sigma$ & Lane 1 & Lane 2 & Lane 3 & Lane 1 & Lane 2 & Lane 3 \\
\hline 1 & 2.11 & 15.36 & 1.75 & 2.37 & 15.00 & 1.88 \\
2 & 2.69 & 17.08 & 2.65 & 2.05 & 14.89 & 1.65 \\
3 & 3.40 & 19.16 & 3.36 & 2.04 & 15.24 & 1.93 \\
\hline
\end{tabular}

Table II shows the RMSE values averaged over 100 repeated runs for different settings of the parameters. It can be seen from Table II that the estimation errors are larger than their counterparts in Table I. Clearly this is the consequence that the lane change effect was not taken into account in state equation (1). As expected, the estimation for the middle lane, lane 2, is particularly problematic as it was associated with a substantial number of lane changes.

\section{SIMULATION STUDY FOR DENSITY ESTIMATION USING THE TRANSFORMATION}

In this section, simulation experiments will be conducted to examine the approach of transforming the observation equation from the nonlinear form (4) to the linear equation (8), and to investigate its impact on the traffic density estimation. For ease of comparison, the state equation in the comparison will be kept to be the same, i.e. equation (1), so that we can focus on the transformation for the observation equation. Specifically we will consider two state space models: (a) Model I with state equation (1) and the transformed observation equation (8); (b) Model II with state equation (1) and the untransformed observation equation (4).

\section{A. Simulation Description}

From a practical perspective, using state equation (1) in the simulation study implies that we restrict our interests to the scenario where lane change maneuvers are not common in the roadway segments under investigation. Hence, in the experiments conducted in this section, the 'true' traffic densities were simulated with an identity system matrix A. As it was assumed there was no interaction between lanes, we focused on a single lane with two roadway segments in tandem set equal to 400 and 600 meters long respectively. All the other experiment settings were kept to be the same as in the previous section.

\section{B. Simulation Results}

For the second roadway segment with $\lambda=10, \sigma=3$ and $\tau=1$, Fig. 2 (upper) displays the 'true' vehicle counts (broken line) and the corresponding estimates (real line) in one run of the experiment using the transformation method. It can be seen that the transformation method performed well: the estimated 
counts were close to the 'true' values.
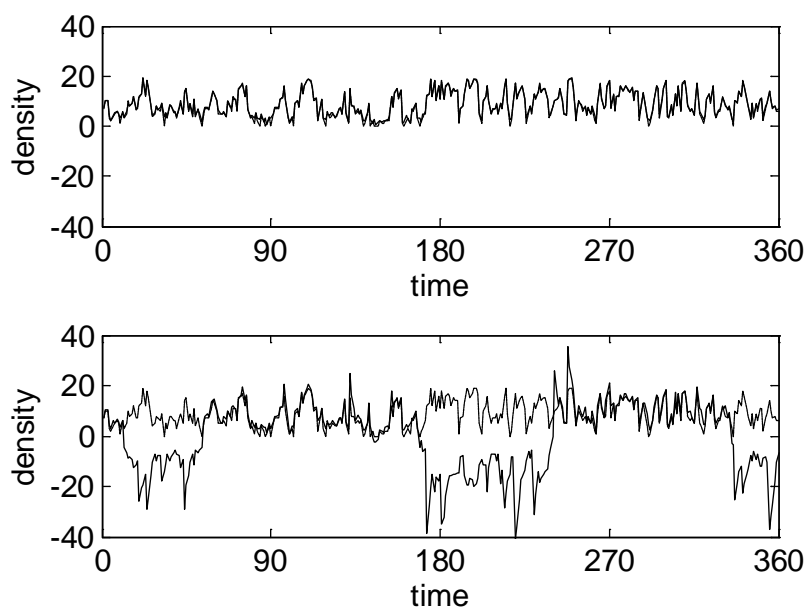

Fig. 2. The 'true' vehicle counts for segment 2 (broken line) and the estimated values (real line) using the transformation approach (upper graph) and using the method in [12] (lower graph).

It is of interest to investigate the impact of the different error parameters $\sigma$ and $\tau$ on the accuracy of estimation. Table III displays RMSE values for different settings using the transformation approach. It can be seen that overall the estimated traffic counts were quite accurate for small $\sigma$ and $\tau$. When $\sigma$ and $\tau$ became large, the average estimation error increased but still was within an acceptable level.

Table III

Average RMSEs over 100 simulation runs for two roadway segments with $\lambda=10$ using the transformation approach

\begin{tabular}{ccccc}
\hline & Segment & $\tau=1$ & $\tau=2$ & $\tau=3$ \\
\hline$\sigma=1$ & 1 & 0.64 & 0.97 & 1.27 \\
& 2 & 0.97 & 1.44 & 1.88 \\
$\sigma=2$ & 1 & 0.62 & 0.98 & 1.29 \\
& 2 & 0.91 & 1.44 & 1.88 \\
$\sigma=3$ & 1 & 0.62 & 0.96 & 1.28 \\
& 2 & 0.88 & 1.39 & 1.87 \\
\hline
\end{tabular}

\section{Comparison with the Existing Method}

Next the performance of the transformation approach will be compared with that used in [12] where no transformation is applied, i.e. vehicle counts are estimated using the extended Kalman filter. Table IV displays the RMSE values for different parameter settings.

Table IV

Average RMSEs over 100 simulation runs for two roadway segments with $\lambda=10$ using the method in [12]

\begin{tabular}{ccccc}
\hline & Segment & $\tau=1$ & $\tau=2$ & $\tau=3$ \\
\hline$\sigma=1$ & 1 & 0.94 & 1.15 & 1.41 \\
& 2 & 1.08 & 1.51 & 1.99 \\
$\sigma=2$ & 1 & 4.66 & 3.62 & 3.21 \\
& 2 & 4.07 & 3.66 & 3.40 \\
$\sigma=3$ & 1 & 11.90 & 7.84 & 6.62 \\
& 2 & 9.03 & 7.31 & 6.82 \\
\hline
\end{tabular}

It can be seen that overall the estimation errors in Table IV were larger than their counterparts in Table III, in particular when the measurement errors were not small. This is not surprising. As pointed out earlier, the method in [12] is an approximate method. In the conducted simulation experiments, it was not unusual that it completely broke down (i.e. greatly deviated from the trajectory of actual vehicular density).

To have a closer look at the performance of the method used in [12], the simulated traffic data used in the upper graph of Fig. 2 were re-analyzed using the method in [12]. The results are displayed in the lower graph of Fig. 2. It can be seen that the performance of the method in [12] was not stable: it can perform well but also can be completely off the track. Overall it was not robust against perturbations. Presumably this was the consequence of the linearization in the extended Kalman filter.

\section{A REAL TRAFFIC STUDY}

In this section, we use real traffic data to illustrate the developed state space model.

The real traffic data measured by two loop detector stations were collected on a normal weekday, Wednesday $13^{\text {rd }}$ June 2007. The loop detector stations are located near Seattle with three lanes in both the westbound and eastbound directions. For illustration purposes, we focused on the westbound lanes only for the time period from 16:00 p.m. to 19:00 p.m.
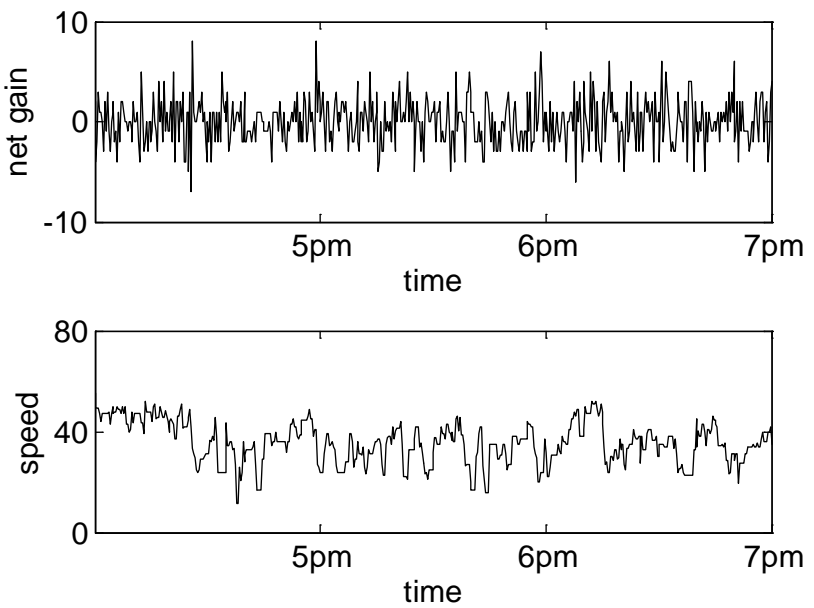

Fig. 3. The net gains of vehicles entering and leaving the roadway segment (upper) and the speed measurements (lower) in the middle lane of the roadway segment.

The segment of interest is defined by the two loop detector stations. The analysis was based on the net gains $\mathbf{w}(t)$ of vehicles entering and leaving the roadway segment and the corresponding speed measurements $\mathbf{v}(t)$. The upper and lower graphs of Fig. 3 display the net gains and the corresponding speed measurements in the middle lane. It can be seen that the traffic condition was stable during the time period of interest. The net gains fluctuated around 0 and the vehicle speed was stable at a relatively low level. The pattern for the innermost 
and outermost lanes (not shown here) is similar.

In the analysis, the data collected on $13^{\text {rd }}$ June 2007 were treated as modeling data upon which the system matrix $\mathbf{A}$ in the state equation (5) was identified using MATLAB Systems Identification toolbox, as shown below:

$$
\mathbf{A}=\left[\begin{array}{ccc}
0.77 & 0.22 & 0 \\
0.23 & 0.62 & 0.17 \\
0 & 0.16 & 0.83
\end{array}\right]
$$

Clearly, for all three lanes there were a considerable proportion of vehicles changing their lanes. For lane 2, for example, there were about $22 \%$ of vehicles in lane 2 changing to lane 1 , and about $16 \%$ of vehicles changing to lane 3 . Hence the lane change effect was not ignorable.

To illustrate the developed method, we also collected the net gain data and speed measurements for the same time period one week later, i.e. Wednesday $20^{\text {th }}$ June 2007. We first assumed that the pattern of lane-change was retained unchanged, as shown in (9). Using the developed state space model with equations (5) and (8), we applied the Kalman filter to estimate traffic densities on $20^{\text {th }}$ June 2007. Here for illustration purposes we followed [12] to set the tuning parameters in the analysis. In practice, these parameters should be carefully tuned in order to achieve the best performance. The estimated traffic densities in the middle lane are displayed in Fig. 4.

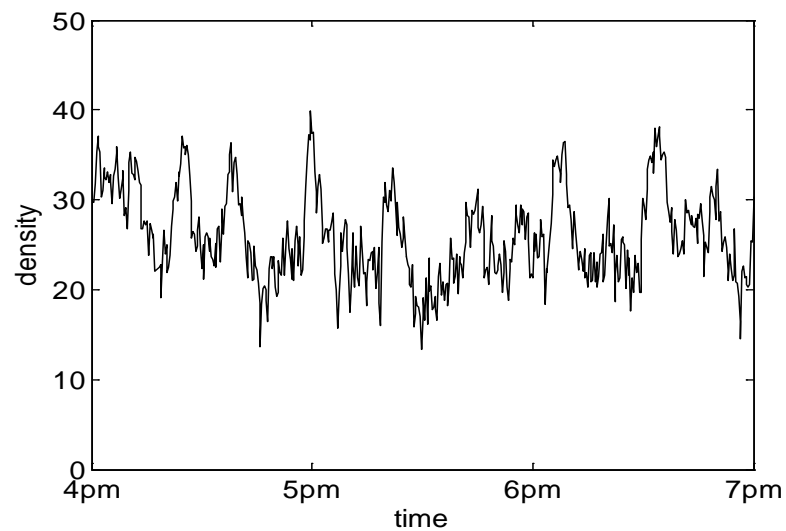

Fig. 4. The estimated traffic densities in the middle lane of the roadway segment using system matrix $\mathbf{A}$ in (9).

To investigate the issue of robustness, we also estimated the system matrix using the data on $20^{\text {th }}$ June 2007 :

$$
\widetilde{\mathbf{A}}=\left[\begin{array}{ccc}
0.79 & 0.20 & 0 \\
0.21 & 0.66 & 0.15 \\
0 & 0.14 & 0.85
\end{array}\right] \text {. }
$$

Due to sampling error, it is not surprising that matrix $\widetilde{\mathbf{A}}$ differs from A given in (9). However, it is also clear that the difference is small and the general pattern is kept unchanged. This suggests that the lane change behavior was more or less stable and did not change much.

We then applied the Kalman filter with matrix $\widetilde{\mathbf{A}}$ to estimate traffic densities for the same data. It turned out that the estimated traffic densities were close to that obtained earlier using matrix $\mathbf{A}$ in (9): the RMSE between them is 0.24, i.e. less than a quarter of one vehicle. Hence, the approach is not sensitive to small changes in the entries of the system matrix.

\section{CONCLUSIONS AND DISCUSSION}

In this paper, we have investigated traffic flow modeling via the approach of state space model, and the application of Kalman filter to roadway traffic density estimation. We have used a Markov chain to describe lane change behavior so that the state equation can better reflect the movements of vehicles between lanes. In addition, in order to avoid the approximation in the extended Kalman filter, we have investigated a transformation approach which transforms the nonlinear observation equation to a linear form. The performance of the developed state space model was investigated in the simulation studies. It is shown that it has substantially increased the accuracy of the traffic density estimation.

The developed approach has important practical implications. In practice, loop detectors are widely deployed in strategic roadway networks so potentially the developed methods can be widely applied to provide an effective approach to traffic surveillance. The estimated traffic density can facilitate traffic management of networks, and also provide inputs for both long-term transport planning and short-term traffic control.

It should be noted that the developed state space model in this paper has some limitations. First, it is assumed that relatively dense loop detector stations are deployed (for instance, they are about 500 meters to 1500 meters apart) to provide the required data for traffic density estimation. In practice when this is not the case, the develop approach must be used with care: the estimation errors can become larger when a segment is longer. Secondly, it is assumed that the traffic condition is stable so that the probabilities of lane change retain approximately constant. The assumption of a time-invariant system could go wrong in reality. This is because state equation (5) considered in this paper is simply an approximation of a more general time-varying system. Technically one possible approach to addressing this issue is to incorporate an adaptive algorithm to online update the coefficients of the system matrix in state equation (5). This approach will be explored in our future research.

\section{ACKNOWLEDGMENT}

The authors would like to thank the four reviewers for their helpful comments on the earlier versions of this paper.

\section{REFERENCES}

[1] T. Z. Qiu, X. Y. Lu, A. H. F. Chow, and S. Shladover, "Real-time density estimation on freeway with loop detector and probe data," Journal of the Transportation Research Board, vol. 2178, pp. 21-28, Jan. 2011.

[2] $\mathrm{B}$. $\mathrm{Li}$, "On the recursive estimation of vehicular speed using data from a single inductance loop detector: a Bayesian approach," Transportation Research Part B, vol. 43, pp. 391-402, May 2009. 
[3] B. Li, "Bayesian inference for vehicle speed and vehicle length using dual-loop detector data," Transportation Research Part B, vol. 44, pp. 108-119, Jan. 2010

[4] B. D. Greenshields, "The photographic method of studying traffic behaviour," in Proc. 13th Annu. Meeting of the Highway Research Board, 1933.

[5] B. D. Greenshields, "A study of highway capacity," in Proc. Highway Research Record, Washington, vol. 14, pp. 448-477, 1935.

[6] S. Kim and J.-H. Kim, "Adaptive fuzzy-network-based C-measure mapmatching algorithm for car navigation system," IEEE Trans. on Industrial Electronics, vol. 48, pp. 432-441, Apr. 2001.

[7] T. Bucher, C. Curio, J. Edelbrunner, C. Igel, D. Kastrup, I. Leefken, G. Lorenz, A. Steinhage, and W. von Seelen, "Image processing and behavior planning for intelligent vehicles," IEEE Trans. on Industrial Electronics, vol. 50, pp. 62-75, Feb. 2003.

[8] M. Wada, K. S. Yoon, and H. Hashimoto, "Development of advanced parking assistance system," IEEE Trans. on Industrial Electronics, vol. 50, pp. 4-17, Feb. 2003.

[9] B.-F. Wu, C.-T. Lin, and Y.-L. Chen, "Dynamic calibration and occlusion handling algorithms for lane tracking," IEEE Trans. on Industrial Electronics, vol. 56, pp. 1757-1773, May 2009.

[10] E. Manla, A. Nasiri, C. H. Rentel, and M. Hughes, "Modeling of zinc bromide energy storage for vehicular applications," IEEE Trans. on Industrial Electronics, vol. 57, pp. 624-632, Feb. 2010.

[11] M. W. Szeto, and D. C. Gazis, "Application of Kalman filtering to the surveillance and control of traffic systems," Transportation Science, vol. 6, pp. 419-439. Nov. 1972.

[12] D. Gazis, and C. Liu, "Kalman filtering estimation of traffic counts for two network links in tandem," Transportation Research Part B, vol. 37, pp. 737-745, Sept. 2003.

[13] Y. Wang, and M. Papageorgiou, "Real-time freeway traffic state estimation based on extended Kalman filter: A general approach," Transportation Research Part B, vol. 39, pp. 141-167, Feb. 2005.

[14] X. Sun, L. Muñoz, and R. Horowitz, "Mixture Kalman filter based highway congestion mode and vehicle density estimator and its application,” in Proc. 2004 American Control Conference, Jun. 2004, pp. 2098-2103.

[15] G. Vigos, M. Papageorgiou, and Y. Wang, "Real-time estimation of vehicle-count within signalized links," Transportation Research Part C, vol. 16, pp. 18-35, Feb. 2008.

[16] P. G. Gipps, "A model for the structure of lane-changing decisions", Transportation Research Part B, vol. 20, pp. 403-414, Oct. 1986.

[17] [17] J.-B. Sheu, "A stochastic modeling approach to dynamic prediction of section-wide inter-lane and intra-lane traffic variables using point detector data," Transportation Research Part A, vol. 33, pp. 79-100, Feb. 1999.

[18] J. S. Drake, J. L. Schofer, and A. D. May. "A statistical analysis of speed density hypotheses," Highway Research Record, vol. 154, pp. 53-87, 1967.

[19] D. Simon, Optimal State Estimation: Kalman, H Infinity, and Nonlinear Approaches. New York: Wiley, 2006.

[20] R. Muradore and P. Fiorini. "A PLS-Based statistical approach for fault detection and isolation of robotic manipulators”, IEEE Trans. on Industrial Electronics (in press).

[21] R. Toscano, and P. Lyonnet. "A Kalman optimization approach for solving some industrial electronics problems," IEEE Trans. on Industrial Electronics (in press).

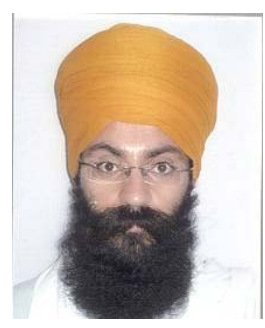

Karandeep Singh received the B.Sc. (Hons) in Mathematics and M.Sc. in Operational Research from the University of Delhi, India, in 2005 and 2007, respectively, and is currently working toward the Ph.D. degree in the School of Business and Economics, Loughborough University, UK. From April 2007 to July 2008, he was working as a Search Engine Optimiser in New Delhi, India. His current research interests include statistical modelling, Markov theory, control theory applications, multilevel modelling for transportation management and health care systems.

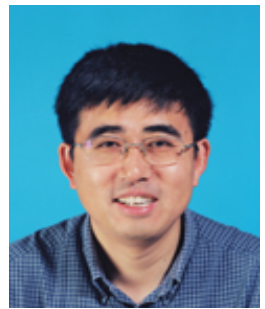

Baibing Li received the B.Sc. degree from Yunnan University, and the M.Sc. degrees from Shanghai Jiaotong University and Vrije Universiteit Brussel respectively. In 1991 he received the Ph.D. degree from Management School, Shanghai Jiaotong University, Shanghai, China. He worked as a Postdoctor and Research Fellow with Katholieke Universiteit Leuven, and as a Research Associate with Newcastle University. In 2001, he was appointed Lecturer at Newcastle University. He moved to the School of Business \& Economics, Loughborough University, Leicestershire, UK, as Lecturer in 2004 and subsequently was appointed as Reader in 2007 and Professor in 2011. In recent years much of his work has involved transport and traffic management such as transportation demand analysis, travel behavior modeling, and intelligent transportation systems. His current research interests are Bayesian statistical modeling and forecasting for non-Gaussian, dynamic, nonlinear problems in various management areas.

Prof. Li is a member of Royal Statistical Society. 